\title{
SISTEMA EN TIEMPO REAL PARA EL MONITOREO DE VARIABLES MÉDICAS EN PACIENTES HOSPITALIZADAS CON REDES WSN
}

\author{
REAL-TIME SYSTEM FOR MONITORING MEDICAL VARIABLES \\ WITH WSN NETWORKS IN HOSPITALIZED PATIENTS
}

\author{
${ }^{1}$ Sixto Enrique Campaña Bastidas, ${ }^{2}$ Adriana Aguirre Cabrera, \\ ${ }^{3}$ Harold Emilio Cabrera Meza, ${ }^{4}$ Alvaro José Cervelion \\ Universidad Nacional Abierta y a Distancia-Unad-Ecbti. Pasto-Colombia
}

\section{RESUMEN}

Este artículo presenta los resultados de la investigación PIE 03-15 aprobada por el Comité de Investigaciones de la Ecbti de la unad en el año 2015, la cual consistió en un estudio de aplicación de un sistema en tiempo real para el monitoreo de variables médicas, en pacientes hospitalizados. Para el caso específico, se tomó como referencia una población de personas con patología de preeclampsia. La investigación en referencia partió de la descripción de la población seleccionada, así como de la patología asociada; luego, se presentaron los requerimientos técnicos y lógicos del sistema propuesto y se finalizó con los resultados que arrojó el desarrollo del sistema. Este estudio es un inicio en la aplicación y desarrollo de dispositivos y sistemas para la captura de señales biomédicas utilizando tecnologías emergentes, con software y hardware a bajo costo.

Palabras clave: comunicación biomédica, preeclancia,procesamiento de señales biomédicas, redes de área personal, redes de sensores inalámbricos, redes de sewnsores del cuerpo, sistema software.

\section{Abstract}

This article presents the results of the research onapplication of a real-time system for monitoring of medical variables in hospitalized patients. For this case, a population with pathology of pre-eclampsia was taken as reference. The research began with the description of the selected population and the associated pathology; then, the technical and logical requirements of the proposed system were presented and it was finalized with the results obtained in the development of the system. This study is a start in the application and development of devices and systems for capturing biomedical signals using emerging technologies, with low-cost software and hardware.

\footnotetext{
${ }^{1}$ sixto.campanalunad.edu.co, orcid.org/0000-0001-9937-2784

2 adriana.aguirre@unad.edu.co, orcid.org/0000-0002-8625-3150

${ }^{3}$ harold.cabrera@unad.edu.co, orcid.org/0000-0002-7904-1774

${ }^{4}$ alvaro.cervelion@unad.edu.co, orcid.org/0000-0001-5823-8992
} 
Keywords: biomedical communication, biomedical signal processing, body sensor networks, personal area networks, preeclampsia, software system, wireless sensor networks.

\section{INTRODUCCIÓN}

Las redes de sensores inalámbricas (WSN) son una tendencia tecnológica que cobra cada día mayor importancia en todos los aspectos que atañen a los seres humanos; un ejemplo claro se tiene en el campo de la salud, en el que su aplicación permite un mayor control de enfermedades, seguimiento a patologías especiales y monitoreo a pacientes, entre otras posibilidades. En determinados casos, su uso puede prevenir desenlaces fatales y su ayuda es determinante para el seguimiento que hacen los médicos a un paciente específico.

En la aplicación que se expone en este artículo y que relaciona a las WSN y la salud, se presenta el seguimiento que se hace a personas que pueden llegar a padecer una patología denominada preeclampsia, la cual se define como una afectación médica que, por lo general, afecta a las mujeres embarazadas (Rojas, 2009) lo que demanda un seguimiento riguroso de la presión arterial y de la cantidad de proteína en la orina de las pacientes asociadas a esta enfermedad, sobre todo luego de las 20 semanas de embarazo.

El estudio inició con la caracterización de la población objeto de investigación. Acorde con los resultados obtenidos, se diseñó el sistema de monitoreo con WSN, para posteriormente desarrollarlo, incluyendo en este último, aspectos como características de los sensores que se deben utilizar y, que, a nivel de esta investigación, se proponen como prototipo; este podrá ser implementado y evaluado en una segunda fase investigativa.

El artículo se ha dividido en cuatro secciones, incluida la introducción, los cuales relacionan los siguientes temas: en la sección II, correspondiente al desarrollo del contenido, se explica el proceso de caracterización de la población objeto de estudio y el diseño del sistema de monitoreo propuesto con WSN; el objetivo principal, es contextualizar al lector acerca de la patología que se ha tomado como caso de estudio y la forma en que la misma puede ser monitoreada por tecnologías WSN. En la sección III, se abordan los resultados y se presenta el desarrollo del sistema de monitoreo propuesto a nivel de software y hardware; en esta sección, se detalla la forma en que el sistema funciona lógicamente, las interfaces creadas y los dispositivos sensores necesarios para la aplicación del sistema propuesto. Finalmente, en la sección IV se presentan las conclusiones y los trabajos futuros que se pueden desarrollar a partir de esta investigación.

\section{Desarrollo de contenido}

En este capítulo se describe la caracterización de la población objeto de estudio; así mismo, se hace un breve estado del arte y se propone el diseño preliminar del sistema de monitoreo con tecnologías WSN. Estos elementos permiten al lector contextualizarse acerca de la aplicabilidad de las WSN en el campo de la salud y la forma en que este tipo de tecnologías pueden dar soluciones a situaciones que día a día viven las personas.

\subsection{Caracterización de pacientes con preeclampsia}

La preeclampsia es un desorden multisistémico, caracterizado por la presencia de hipertensión y proteinuria (exceso de proteína en la orina) que se presenta en mujeres en estado de embarazo, generalmente después de las 20 semanas; esto conlleva a cambios patológicos que afectan órganos como la placenta, el riñón, hígado y cerebro, entre otros. De las complicaciones graves del embarazo, esta es la más común y temida, por lo que se debe diagnosticar y tratar rápidamente, ya que en casos severos pone en peligro la vida de la madre y el feto (Rojas, 2009). Aún no se conoce con certeza la causa exacta de la preeclampsia (Luna, 2009), pero hay ciertos factores que pueden provocar esta complicación en el embarazo. En este estudio se ha realizado una 
caracterización de pacientes con esta patología (tomando datos del Hospital Departamental de Nariño), con el fin de evidenciar las posibles causas y las variables médicas más comunes.

\subsubsection{Tipos de preeclampsia}

La hipertensión en mujeres gestantes se denomina clásicamente como preeclampsia, la cual se define como leve o severa, de acuerdo con la siguiente condición clínica (Hipertensión y embarazo: preclampsia s.f.):

Preeclampsia leve: hipertensión de al menos 140/90 $\mathrm{mmHg}$ en dos ocasiones, con seis horas de diferencia después de la semana 20 de embarazo y proteinuria significativa mayor a $300 \mathrm{mg}$ en 24 horas, edema moderado y volumen urinario en 24 horas mayor a $500 \mathrm{ml}$.

Preeclampsia severa: tensión arterial mayor que $160 / 90 \mathrm{mmHg}$ en dos ocasiones con seis horas de diferencia después de la semana 20 de gestación; tensión arterial sistólica mayor de $160 \mathrm{mmHg}$ sobre el valor basal; tensión arterial diastólica mayor de $90 \mathrm{mmHg}$ sobre el valor basal; proteinuria mayor de $5 \mathrm{~g}$ en 24 horas, edema masivo, oliguria (menor de $400 \mathrm{ml}$ en 24 hs), síntomas sistémicos como edema de pulmón, cefalea, alteraciones visuales, dolor en hipocondrio derecho, elevación de las enzimas hepáticas o trombocitopenia. La aparición de una convulsión en pacientes con signos y síntomas de preeclampsia sin datos de traumatismos o enfermedad neurológica identifica a la paciente con eclampsia.

\subsubsection{Variable, caracterización}

Gracias a los datos proporcionados por el Hospital Universitario Departamental de Nariño, se identificaron variables de tipo demográfico y médico de cada una de las pacientes. Los datos demográficos hacen referencia a la información general de cada paciente y se definen generalmente como variables de tipo categórico. Las variables médicas son resultado del examen de valoración o pruebas de laboratorio y pueden ser de tipo numérico o categórico. Para el estudio realizado no se tuvieron en cuenta las variables demográficas, dado que no permiten hacer generalizaciones por tratarse de datos muy descriptivos de cada paciente. Con respecto a las variables médicas, se consideró que todas eran importantes para la definición del perfil de las pacientes con preeclampsia, quedando el bloque de variables que se presenta en la Tabla 1 :

TABLA 1.

Variables caracterización pacientes

\begin{tabular}{|c|c|c|}
\hline Variable & $\begin{array}{l}\text { Tipo de } \\
\text { Variable }\end{array}$ & $\begin{array}{l}\text { Posibles valores } \\
\text { en los registros }\end{array}$ \\
\hline Edad & Numérica & $14=<\mathrm{Edad}=<46$ \\
\hline Año & Numérica & $2010=<$ año $=<2014$ \\
\hline Régimen & Categórica & Subsidiado o contributivo. \\
\hline Departamento & Categórica & Narińo o Putumayo. \\
\hline Municipio & Categórica & $\begin{array}{l}\text { Municipios de Nariño o } \\
\text { Putumayo. }\end{array}$ \\
\hline Estrato & Numérica & $1=<$ estrato $=<5$ \\
\hline Etnia & Categórica & Indígena, afro o mestizo. \\
\hline Estado civil & Categórica & Casada, soltera o unión libre. \\
\hline IMC & Numérica & $22=<\mathrm{IMC}=<38$ \\
\hline $\begin{array}{l}\text { Periodo de } \\
\text { gestación }\end{array}$ & Categórica & $\begin{array}{l}\text { Puede tomar los valores de } 2 \mathrm{~T} \\
\text { o } 3 \mathrm{~T} \text {, que significa segundo } \\
\text { trimestre o tercer trimestre, } \\
\text { respectivamente. }\end{array}$ \\
\hline PA_sistólica & Numérica & $138=<$ PA_sistólica $=<195$ \\
\hline PA_diastólica & Numérica & $88=<$ PA_diastólica $=<145$ \\
\hline Primigravida & Categórica & Solo hay dos valores: Sí o No \\
\hline Hospitalización & Categórica & Sí o No \\
\hline Proteinuria & Numérica & $\begin{array}{l}\text { Puede ser por debajo de los } 5 \mathrm{~g} \\
\text { o por encima de los } 5 \mathrm{~g} \text { para } \\
\text { preeclampsia moderada o seve- } \\
\text { ra, respectivamente. }\end{array}$ \\
\hline $\begin{array}{l}\text { Antecedentes } \\
\text { familiares }\end{array}$ & Categórica & $\begin{array}{l}\text { No, primer grado y segun- } \\
\text { do_grado }\end{array}$ \\
\hline DXPRIN & Categórica & $\begin{array}{l}140 \text { preeclampsia moderada, } \\
141 \text { preeclampsia severa o } 149 \\
\text { preeclampsia no determinada }\end{array}$ \\
\hline Temperatura & Numérica & $>38^{\circ} \mathrm{Co}<36^{\circ} \mathrm{C}$. \\
\hline
\end{tabular}

El estudio se realizó con las variables antes mencionadas y se aplicó a 1.544 registros que fueron suministrados por el Área de Estadística del Hospital 
Departamental de Nariño, correspondientes al periodo comprendido entre el año 2010 hasta el año 2014.

\subsubsection{Metodología aplicada para la caracteriza-} ción Se aplicó la metodología Crisp-DM (Cross - Industry Standard Processfor Data Mining) (Moine, 2011), común en estudios de minería de datos, la cual permitió la caracterización de las pacientes con preeclampsia en el departamento de Nariño. Esta metodología estructura el proceso en seis fases: comprensión del negocio, comprensión de los datos, preparación de los datos, modelado, evaluación e implantación (Moine, 2009). La metodología contempla estos pasos jerárquicos y específicos que permiten el desarrollo óptimo de una propuesta que pueda dar solución a un problema de información y que aparentemente no está relacionada.

Para la obtención de los datos finales, análisis y caracterización de las pacientes objeto de estudio, se utilizó una herramienta de software denominada Weka Knowledge Flow (Waikato Environment for Knowledge Analysis) (Witten \& Eibe, 2005) herramienta de libre distribución para diferentes sistemas operativos.

2.2.4. Resultados con Weka Con el paquete de software elegido (Weka), se identificó la tarea de minería de datos (árboles de clasificación), la cual se utilizó para organizar la información recolectada de las variables seleccionadas en los registros suministrados por el hospital. En este contexto se analizó que el estudio tenía una gran mayoría de variables tipo categórico con respecto a las numéricas; por ello y acorde con las herramientas utilizadas, se decidió que una buena alternativa para realizar el proceso de segmentación de la información, era el uso de árboles de clasificación. En Weka (Witten \& Eibe, 2005) se tiene una colección de herramientas de visualización y algoritmos para análisis de datos y modelado predictivo, unidos a una interfaz gráfica de usuario para acceder fácilmente a sus funcionalidades.

Para el modelado, se trabajó con la técnica EM, con el fin de que la herramienta Weka, mediante la técnica seleccionada, determinara el número de cluster para construir el perfil de las pacientes con preeclampsia; de la misma manera, se aplicaron modelos estadísticos básicos.
En el análisis realizado, mediante el uso de la técnica de minería de datos (EM), se identificaron siete (7) clusters, los cuales se validaron por medio de la técnica de clasificación, Multilayer Perceptron (Moine, 2011), en la que se encontró que las métricas están dentro de lo esperado y que el porcentaje de error es mínimo comparado con los resultados encontrados.

\subsubsection{Perfil y caracterización de pacientes con preeclampsia}

Los clusters generados con la técnica EM permitieron hacer una relación más directa entre las diferentes variables que componían cada uno de los registros que suministró el Hospital Universitario Departamental de Narińo. De acuerdo con los datos del apartado anterior y con la técnica aplicada, fue factible generar el perfil o caracterización para las pacientes con preeclampsia que son atendidas en el Hospital, el cual se puede observar en la Tabla 2.

TABla 2.

Caracterización de pacientes con preeclampsia

\begin{tabular}{|c|c|}
\hline Diagnóstico más recurrente & Preeclampsia severa \\
\hline Edad promedio pacientes. & 28 años \\
\hline $\begin{array}{l}\text { Muertes pacientes periodo } \\
\text { análisis. }\end{array}$ & 10 \\
\hline IMC promedio. & $30 \mathrm{Kg} / \mathrm{m} 2$ \\
\hline $\begin{array}{l}\text { Periodo de gestación } \\
\text { de mayor riesgo. }\end{array}$ & $\begin{array}{l}\text { Tercer periodo }(3 \mathrm{~T}) \text {, entre el } \\
\text { sexto y noveno mes }\end{array}$ \\
\hline Primigravias en mayor riesgo. & No \\
\hline $\begin{array}{l}\text { Antecedentes familiares } \\
\text { preponderantes. }\end{array}$ & No \\
\hline $\begin{array}{l}\text { Ciudad con más casos } \\
\text { de preeclampsia. }\end{array}$ & Pasto \\
\hline Estratos con más casos. & 1 y 2 \\
\hline Estado civil. & $\begin{array}{l}\text { Esta enfermedad afecta a } \\
\text { todas las mujeres por igual } \\
\text { con respecto a este tipo de } \\
\text { variable; sean casadas, sol- } \\
\text { teras o en unión libre, todas } \\
\text { son propensas a adquirir el } \\
\text { padecimiento. Hay una ligera } \\
\text { tendencia hacia las mujeres } \\
\text { que viven en unión libre. }\end{array}$ \\
\hline
\end{tabular}


De acuerdo con lo anterior y al identificar que la patología más recurrente fue la de preeclampsia severa, se dedujo, acorde con las características de la misma, que las variables médicas que se deben analizar y a las que se debe hacer seguimiento mediante un sistema de monitoreo con WSN, son las que corresponden a la presión arterial, que se deriva de las variables PA_sistólica y PA_diastólica, como también a la proteinuria, las cuales se enumeran con respecto a los valores de riesgo en la tabla 3.

TABLA 3.

Variables de riesgo de la preeclampsia

\begin{tabular}{|l|l|l|}
\hline \multicolumn{1}{|c|}{ Variable } & \multicolumn{1}{|c|}{ Descripción } & \multicolumn{1}{c|}{ Valores de riesgo } \\
\hline PA_sistólica & $\begin{array}{l}\text { Numérica, presión } \\
\text { arterial. }\end{array}$ & Entre 120 y $210 \mathrm{mmHg}$ \\
\hline PA_diastólica & $\begin{array}{l}\text { Numérica, presión } \\
\text { arterial. }\end{array}$ & Entre 80 y $145 \mathrm{mmHg}$ \\
\hline Proteinuria & Numérica & Entre $2 \mathrm{~g} / 24 \mathrm{~h}$ y $5 \mathrm{~g} / 24 \mathrm{~h}$ \\
\hline
\end{tabular}

Las variables relacionadas en la Tabla 3, que corresponden a la presión arterial y la proteinuria, permiten identificar los principales síntomas de preeclampsia. Dado el riesgo que estos representan para las pacientes gestantes y el feto, estas, generalmente, se deben hospitalizar con el fin de seguir los procedimientos necesarios, tales como, un monitoreo permanente y una toma de temperatura cada hora, en casos graves.

\section{Estado del arte y diseño del sistema de monitoreo}

Una vez se ha caracterizado la patología elegida como caso de estudio en el sistema de monitoreo propuesto, en esta parte del artículo se contextualiza el estudio, se describe un breve estado del arte y se formula el diseño del sistema.

3.1. Estado del arte: para iniciar con la descripción de los trabajos previos con respecto al tema de investigación, es preciso definir el concepto de WSN. Según Akyildiz et al. (2002), WSN es una agrupación de múltiples sensores pequeños, dispersos en un determinado espacio con un fin específico que se caracterizan por ser redes de bajo costo, con poco consumo de energía y que transmiten señales a cortas distancias. Teniendo en cuenta el concepto anterior y la caracterización antes descrita, se puede ubicar esta investigación en un trabajo aplicado que va dirigido a pacientes con preeclampsia, en el que se busca monitorear principalmente las variables médicas relacionadas con la presión arterial.

Es importante mencionar que no se encontraron trabajos relacionados con aplicaciones tecnológicas que traten directamente la preeclampsia asociada a WSN; pero sí algunas aplicaciones que tienen similitud al trabajar con señales biomédicas. A continuación, se hace referencia a algunos estudios importantes.

Estudios relacionados directamente con la salud: en Martínez et al. (2008) se propone un estudio que presenta una caracterización de aplicaciones WSN en los sectores médicos, medioambiental, agrícola e industrial; también hace referencia a una aplicación para la detección de arritmias cardiovasculares en pacientes no críticos, para rehabilitación en espacios cerrados, y propone la arquitectura para la red y los nodos que la conforman.

Otro estudio significativo es el que relaciona la plataforma Shimmer de (González, Núnez y García 2009), cuya sigla significa: Sensing Health with Intelligence, Modularity, Mobility, que es una pequeña plataforma de sensores inalámbricos diseñada por Intel para aplicaciones portátiles de cuidado de la salud. Dentro de las aplicaciones estudiadas, es la más completa. Una limitante de la misma, es el costo de la implementación.

En (Kaschel \& Pérez, 2014) se presenta un diseño integrado de vigilancia ubicua de salud en tiempo real, utilizando Wbsn (Redes de Sensores Corporales Inalámbricos) y las nuevas tecnologías como M2M y computación en la nube. La principal característica de este estudio radica en que considera un servidor médico inteligente ubicado en la nube y un dispositivo móvil inteligente que actúa como router y coordinador o concentrador virtual en el lado cliente-paciente; esta solución ha sido diseñada para pacientes que no pueden tolerar interrupciones en su seguimiento médico, para quienes un sistema de monitoreo en tiempo 
real contribuye a mantener sus vidas. El estudio es una propuesta tecnológica para el desarrollo futuro de una solución ubicua.

Otros trabajos relacionados con WSN: en Zen et al. (2011) se define una propuesta de aplicación de WSN que permite la transmisión de datos en tiempo real para la prevención de incendios en edificios. Es una propuesta de simulación que se realizó en el programa NS-2 y se creó para un entorno cerrado. En (Saavedra et al., 2014) se habla sobre el diseño de una WSN para la lectura de la temperatura en un espacio físico concreto; su uso es más de tipo ambiental. En (Morparia et al., 2010) se hace referencia a la aplicación de WSN en sistemas de tiempo real y análisis de interferencias, principalmente. Actualmente, existe una gran producción alrededor del tema, algunos en desarrollo, otros finalizando; lo interesante es resaltar que poco a poco este tipo de tecnologías se van empoderando de necesidades importantes del ser humano y le dan una mano en la solución de sus problemas.

\subsection{Diseño del sistema de monitoreo}

Partiendo de la premisa de que es importante desarrollar tecnologías limpias no invasivas que permitan el monitoreo en tiempo real de pacientes con sintomatología de preeclampsia severa (Hipertensión y embarazo: preclamsia s.f.), se propuso un sistema que mitigue dicha situación, el cual se describe a continuación.

Descripción general del sistema: se plantea para la patología estudiada un sistema basado en sensores biomédicos portátiles de bajo costo, los cuales, en una infraestructura inalámbrica, posibilitan el cuidado inteligente de la presión arterial, principalmente, en mujeres con preeclampsia severa; a su vez, proporciona una vigilancia omnipresente de las mismas, incluso cuando ellas se mueven, lo que genera mejorar la calidad de atención a esta población. La utilización de sensores inalámbricos contribuye a la monitorización automática de la presión arterial y, por ende, al control temprano de la hipertensión y complicación de la enfermedad, en los casos que así lo requieran (Kappiarukudil \& Ramesh 2010). En la Fig. 1 se presenta el diseño general del sistema basado en sensores, en el que se puede evidenciar que el fin del mismo es aprovechar la infraestructura de telecomunicaciones que actualmente se está implementando en el país, haciendo uso de tecnologías inalámbricas (WSN, Wlan, Ethernet $\mathrm{y}$ Wwan).

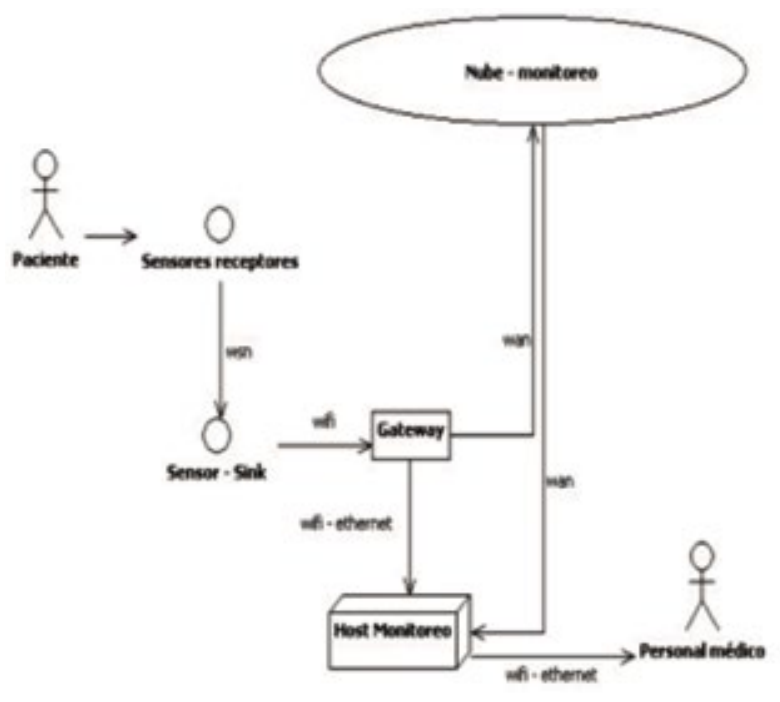

Fig. 1 Diseño general del sistema

Fuente: autores

El diseño propuesto, entonces, se enfoca en un monitoreo permanente de las pacientes con preeclampsia. El sistema se basa en la recolección de datos por medio de sensores ubicados en las pacientes, los cuales transmiten, mediante WSN, los datos al gateway, que los envía a la nube donde el personal médico los consulta para su respectivo análisis y diagnóstico (Abbate, Avvenuti \& Light 2012; Chen et al., 2011; Bravo, Valdez \& Medina, 2015). En esta parte, se analizan las variables relacionadas con la presión arterial de las pacientes.

En el diseño propuesto en la Fig. 1 se observa: sensores WSN, gateway, host de monitoreo y el servicio de recolección de información de los sensores en la nube.

La Fig. 2, por su parte, muestra la interacción entre las variables médicas y la interfaz del sensor en la que se recolectan los datos fisiológicos de las pacientes por medio de los biosensores conectados en su cuerpo, los cuales transmiten en tiempo real los datos al sistema de monitoreo almacenado en la nube. 


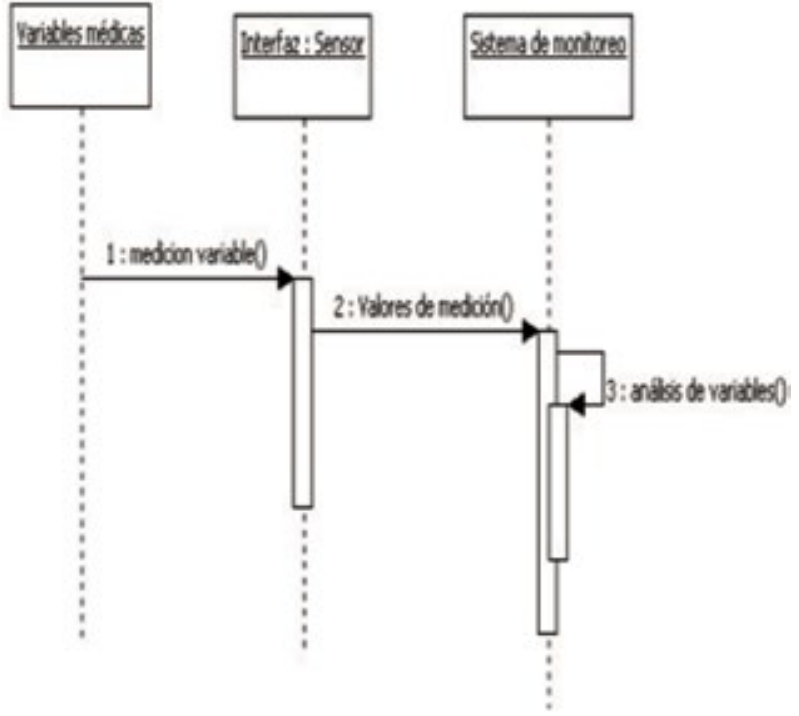

Fig. 2 Interacción variables y sensores

Fuente: autores

Funcionamiento del sistema: el monitoreo de las pacientes con tecnología WSN se implementa mediante los sensores que capturan las variables médicas relacionadas con la preeclampsia. El sistema captura las señales biomédicas y envía las lecturas de las variables de medición de presión arterial a un dispositivo transmisor (gateway), que las retransmite en tiempo real y de manera inalámbrica al host de monitoreo o directamente al sistema instalado en la nube.

El sistema de monitoreo permite comparar las variables de presión arterial para detectar anomalías en la condición clínica de las pacientes, con el fin de alertar al personal médico, vía Web o mediante mensajes a sus teléfonos inteligentes (Shahriyar et al., 2009; Yang \& Huang, 2012).
En términos más específicos, el sistema está compuesto principalmente por un subsistema de información, que funciona mediante sensores conectados al cuerpo de las pacientes con preeclampsia; los sensores de las pacientes se comunican con otros sensores centrales o de control ubicados estratégicamente, también llamados sink, los cuales redirigen la información en tiempo real a la nube o al centro de datos del sistema; esta comunicación se realiza mediante un canal inalámbrico de la frecuencia abierta 2.4 Ghz que trabaja las WSN Fig. 3.

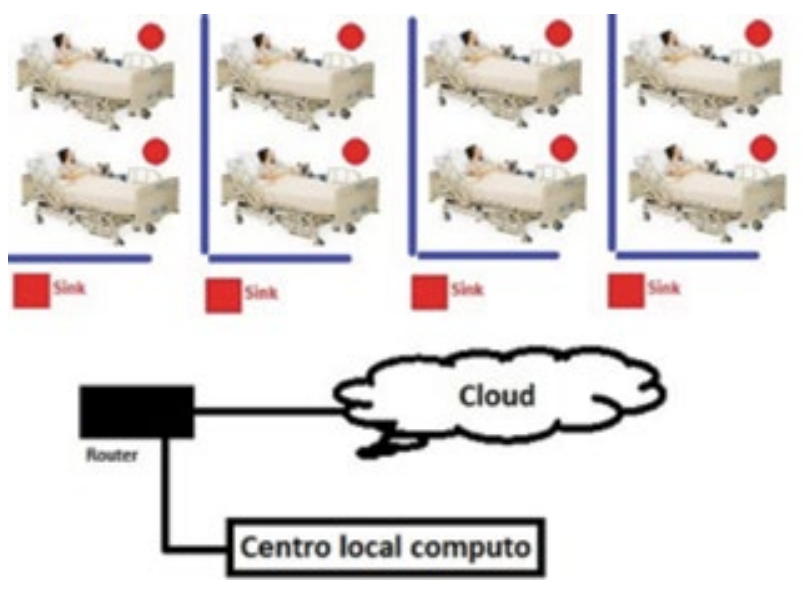

Fig. 3 Escenario general funcionamiento del sistema

\section{Características del sistema:}

Teniendo en cuenta los aspectos antes mencionados relacionados con el diseño y funcionamiento del sistema, en la tabla 4 se enumeran las características que debe poseer el mismo para su implementación. 


\section{TABla 4.}

Característica del sistema

\begin{tabular}{|l|l|}
\hline \multicolumn{1}{|c|}{ Característica } & \multicolumn{1}{c|}{ Descripción } \\
\hline Población objetivo. & $\begin{array}{l}\text { Pacientes con preeclampsia severa hospitalizadas en una } \\
\text { unidad médica de un hospital de tercer nivel. }\end{array}$ \\
\hline $\begin{array}{l}\text { Lectura en tiempo real de } \\
\text { variables biomédicas: presión } \\
\text { arterial y temperatura. }\end{array}$ & $\begin{array}{l}\text { Se utilizarán sensores biomédicos para la lectura de las } \\
\text { variables propuestas, los cuales serán no invasivos. }\end{array}$ \\
\hline $\begin{array}{l}\text { Transmisión de datos en } \\
\text { tiempo real a un centro de } \\
\text { cómputo o nube (cloud). }\end{array}$ & $\begin{array}{l}\text { El sistema transmitirá mediante el Sink, de una WSN, } \\
\text { los datos capturados por los sensores, y, posteriormente, } \\
\text { mediante un gateway se enviarán a un centro de cómputo } \\
\text { o nube para su procesamiento. }\end{array}$ \\
\hline $\begin{array}{l}\text { Generación de alertas de } \\
\text { acuerdo con la información } \\
\text { capturada. }\end{array}$ & $\begin{array}{l}\text { En el centro de cómputo o nube se correrá una aplicación } \\
\text { que evaluará los datos que se carguen y cuando sea necesa- } \\
\text { rio de acuerdo con los datos capturados y comparados con } \\
\text { los mínimos aceptables en el sistema. }\end{array}$ \\
\hline $\begin{array}{l}\text { Consulta en línea de los valo- } \\
\text { res que capturan los sensores. }\end{array}$ & $\begin{array}{l}\text { Se dispondrá de una aplicación Web que permitirá el mo- } \\
\text { nitoreo en tiempo real de las pacientes objeto de estudio } \\
\text { mediante los sensores implantados en las mismas. }\end{array}$ \\
\hline $\begin{array}{l}\text { Generación de reportes } \\
\text { estadísticos }\end{array}$ & $\begin{array}{l}\text { El sistema generará reportes con respecto a la evolución de } \\
\text { la patología (preeclampsia) en las pacientes monitoreadas y } \\
\text { acorde con las alertas generadas y el seguimiento realizado. }\end{array}$ \\
\hline
\end{tabular}

Con las características mencionadas en la tabla

que fueron necesarios para la implementación del 4 se definieron los elementos de hardware y software sistema, tabla 5.

\section{TABla 5.}

Requerimientos del sistema

\begin{tabular}{|l|l|l|}
\hline \multicolumn{1}{|c|}{ Requerimiento } & \multicolumn{1}{|c|}{ Tipo } & \multicolumn{1}{c|}{ Descripción } \\
\hline Sensores biomédicos. & Hardware & $\begin{array}{l}\text { Se implantarán de forma no invasiva dos en cada paciente: uno, para } \\
\text { la lectura de la presión arterial y otro, para la lectura de la temperatu- } \\
\text { ra en tiempo real. }\end{array}$ \\
\hline Sensores sink. & Hardware & $\begin{array}{l}\text { Estos sensores serán los encargados de capturar la información de los } \\
\text { sensores biomédicos; estarán conectados por medio de una WSN. }\end{array}$ \\
\hline Gateway o pasarela. & Hardware & $\begin{array}{l}\text { Equipo que permitirá hacer la transmisión de datos de una WSN a } \\
\text { una WIFI o Ethernet. }\end{array}$ \\
\hline $\begin{array}{l}\text { Centro de cómputo o } \\
\text { nube }(\text { cloud }) .\end{array}$ & $\begin{array}{l}\text { Hardware } \\
\text { y Software }\end{array}$ & $\begin{array}{l}\text { La captura de datos se implementará en una nube de internet, pero } \\
\text { se tendrá un sistema de respaldo local en el hospital mediante una } \\
\text { aplicación Web. }\end{array}$ \\
\hline Sistema de alertas. & Software & $\begin{array}{l}\text { Aplicación Web que permitirá evaluar los datos capturados versus los } \\
\text { mínimos deseados en las pacientes; esta aplicación estará en la nube y } \\
\text { en el centro de datos. }\end{array}$ \\
\hline Sistema de reportes. & Software & $\begin{array}{l}\text { Aplicación Web que generará los reportes estadísticos del seguimiento } \\
\text { a las pacientes con preeclampsia. }\end{array}$ \\
\hline Sistema de consulta. & Software & $\begin{array}{l}\text { Aplicación Web que permitirá hacer seguimiento en línea y en tiempo } \\
\text { real del funcionamiento de los sensores en las pacientes. }\end{array}$ \\
\hline
\end{tabular}


Con los anteriores elementos definidos, en el siguiente capítulo se expone el desarrollo del sistema a nivel de software y hardware, que al momento de finalizar esta investigación estará en fase de prototipo, planeando una segunda fase de implementación.

\section{Resultados y ANÁlisis}

En este capítulo se describen los resultados de la investigación, partiendo de la descripción del prototipo de sensores propuesto para el monitoreo de las variables identificadas en el caso de aplicación de la patología de preeclampsia; luego, se detalla el desarrollo realizado con respecto al software que constituye el sistema de monitoreo y se finaliza con la descripción de los reportes que genera el sistema y los que a futuro se pueden desarrollar.

\subsection{Prototipo de sensores para monitorear a pacientes con preeclampsia}

En el capítulo II se identificó que una de las variables que se debe medir y monitorear de forma periódica en pacientes con preeclampsia, es la presión arterial; por ello, antes de describir los sensores y dispositivos que hacen parte del prototipo, es preciso definir algunos conceptos relacionados.

\subsubsection{Presión arterial}

Se define como la presión hidrostática ejercida por la sangre sobre las arterias como resultado de la contracción del ventrículo izquierdo del corazón (López 2012). Durante cada latido del corazón, la presión sanguínea varía entre una presión sistólica (máxima) y una presión diastólica (mínima) generadas por la acción de bombeo del corazón. La medición de la presión arterial es muy importante, debido a que no solo refleja el funcionamiento del corazón sino de varios órganos importantes como los rińones, hígado, cerebro, entre otros (Chaithanya, Kishore y Srinivasulu 2014). En un adulto sano en reposo, la presión arterial sistólica es de alrededor de $110 \mathrm{mmHg}$ (milímetros de mercurio) y la presión arterial diastólica es de alrededor de 70 mm Hg (López, 2012) en condiciones normales. Valores por encima de $130 / 90 \mathrm{mmHg}$ son indicativos de hipertensión o presión alta y por debajo de 100/60
$\mathrm{mmHg}$ son indicativos de hipotensión o presión baja (Godoy, 2010).

Medición de presión arterial: existen dos métodos para realizar la medición de presión arterial, los cuales se describen a continuación:

Control de presión arterial invasiva: consiste en la medición directa de la presión arterial mediante la inserción de una aguja de cánula en una arteria. Por lo tanto, solo se utiliza en entornos hospitalarios. Este tipo de monitoreo es la forma más precisa de medir la presión arterial y permite la monitorización continua, aunque tiene un alto riesgo de infecciones y complicaciones (Nummelin, 2015).

Control de presión arterial no invasiva: los monitores de presión arterial no invasiva realizan la medición por encima de la piel, utilizando la técnica de auscultación (Riva-Rocci, Korotkoff) con la ayuda de un esfigmomanómetro o manómetro de mercurio o la técnica oscilométrica empleada en los monitores automáticos (Nummelin, 2015), Fig. 4.
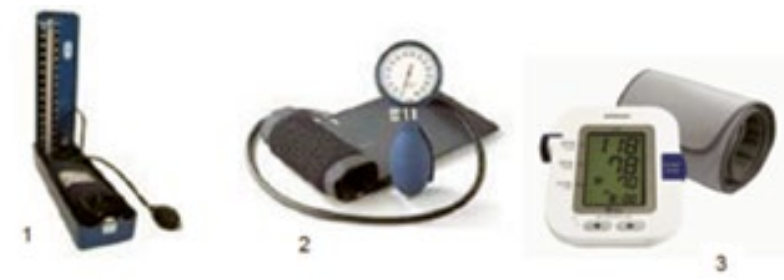

Fig. 4 Manómetro de mercurio (1), esfingomanómetro (2), monitor electrónico (3) (Nummelin 2015)

Fuente: (Nummelin 2015)

\subsubsection{Sensores propuestos para el prototipo}

Los sistemas de control mencionados en el punto 1.2. requieren la utilización de un brazalete que bloquea el flujo de sangre; este es incómodo para el paciente $y$, por tanto, no se puede utilizar de forma continua. Por esta razón, se propone un método para la medición de presión arterial que permita realizar varias mediciones por día con una carga baja de batería para el paciente, apoyados en un sensor electrónico.

El monitor propuesto es un dispositivo portátil, que se constituirá en un sensor de presión, el cual no requiere 
estar conectado a la energía eléctrica, ni de un teléfono inteligente o computadora para funcionar. Le permite al paciente realizar sus propias mediciones de manera sencilla sin que requiera del apoyo de personal médico.

Sensores de presión: este tipo de sensores generan una señal eléctrica proporcional a la presión aplicada sobre él. El sensor de presión se puede clasificar de acuerdo con el tipo específico de medición como presión absoluta, presión diferencial y presión manométrica. El sensor de presión absoluta detecta y mide la presión con referencia a una presión absoluta como se muestra en la Fig. 5. El sensor de presión diferencial realiza la medición en relación a una presión de referencia conocida como se observa en la Fig. 6. Este sensor mide la diferencia entre estas dos presiones, que son la presión aplicada y la presión de referencia. El sensor de presión manométrica opera con el mismo principio que el sensor de presión diferencial, pero detecta y mide la presión con referencia a la presión atmosférica ambiente (ver Fig. 7). Este tipo de sensor de presión tiene una amplia gama de aplicaciones; sin embargo, la medición del mismo no es coherente debido a que la presión de referencia, que es la presión atmosférica, varía de acuerdo con la altitud (Yunus et al., 2015).

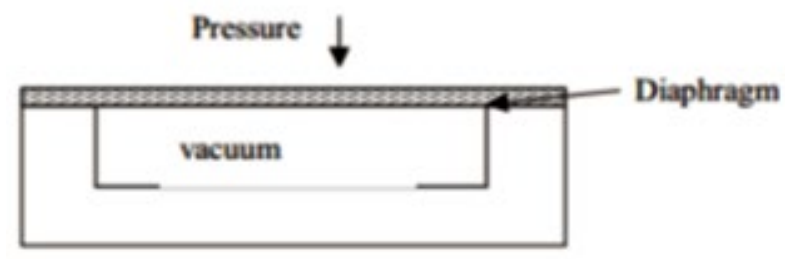

Fig. 5 Sensor de presión absoluta.

Fuente: (Yunus et al. 2015)

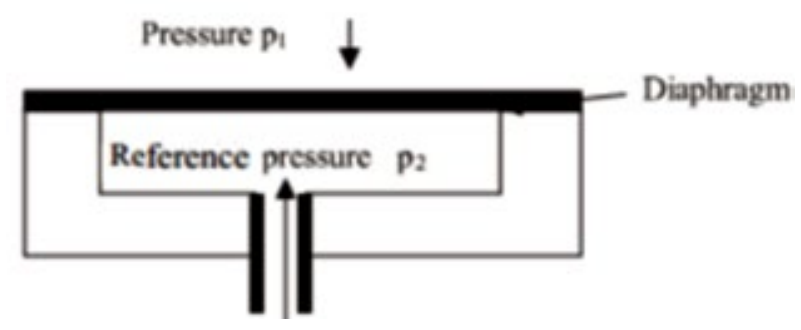

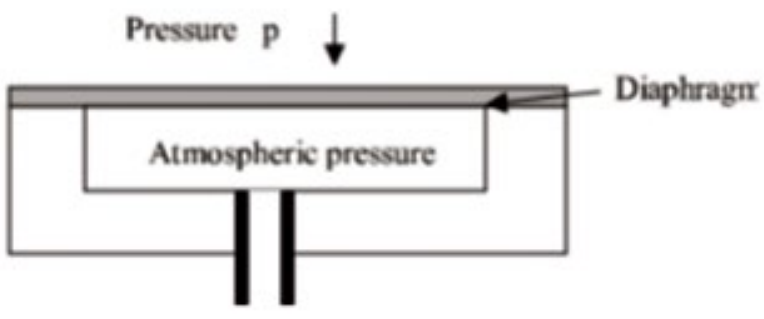

Fig. 7 Sensor de presión atmosférica.

Fuente: (Yunus et al., 2015)

Los sensores de presión también se pueden clasificar de acuerdo con su elemento de detección utilizado. De esta manera, se clasifican en sensores piezorresistivos, piezoeléctricos y capacitivos.

El sensor de presión piezorresistivo Fig. 8, utiliza el efecto piezorresistivo para detectar la presión aplicada. En otras palabras, el sensor cambiará en su resistencia cuando se somete a una presión (Rathore \& Panvar, 2013). El sensor de presión piezoeléctrico (Fig. 9) cuando detecta una presión aplicada genera un cambio de potencial eléctrico a su salida (Mohammadi et al., 2009). El sensor de presión capacitivo Fig. 10, cambiará su capacitancia proporcional a la presión aplicada (Shahiri, Ganji y Sabbaghi, 2012).

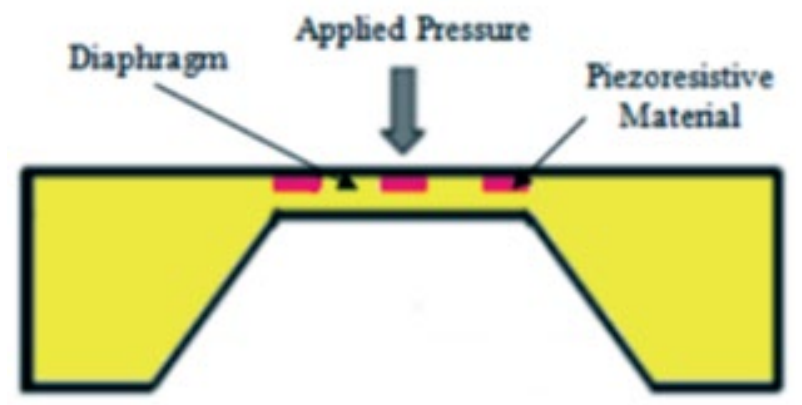

Fig. 8 Sensor de presión Piezorresistivo Fuente: (Nurul et al. 2015)

Fig. 6 Sensor de presión diferencial

Fuente: (Yunus et al. 2015) 


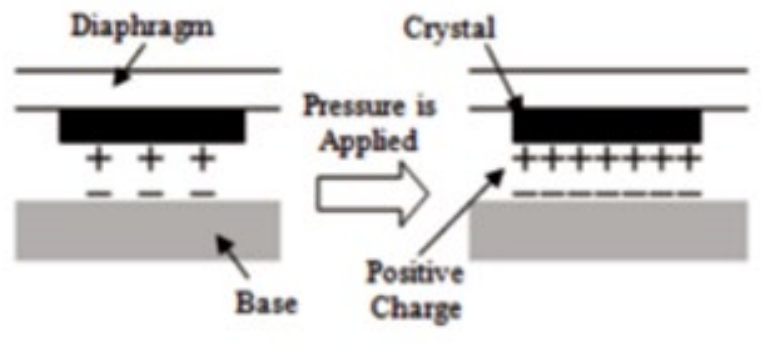

Fig 9. Sensor de presión Piezoeléctricos Fuente: (Nurul et al., 2015)
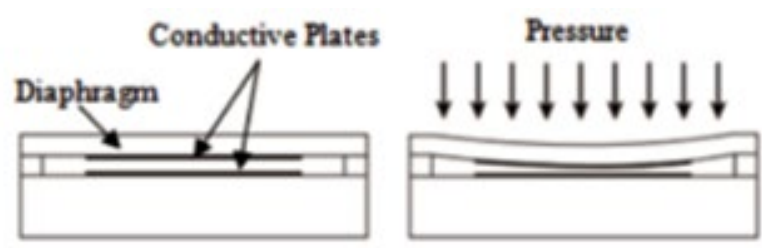

Fig. 10 Sensor de presión capacitivo (Yunus et al., 2015)

Monitor de presión arterial: el dispositivo propuesto permite realizar mediciones de presión arterial por vía percutánea. Se mide el desplazamiento de una superficie causada por el movimiento de una pared del vaso sanguíneo, debido a su sobrepresión en el interior, como se muestra la Fig.11. Este método se conoce como tonometría; cuando el sensor de presión se ubica en el lugar correcto de la superficie, este desplazamiento puede ser detectado y entrega una seńal proporcional a la presión de la sangre dentro del vaso sanguíneo (Kirstein et al., 2005).

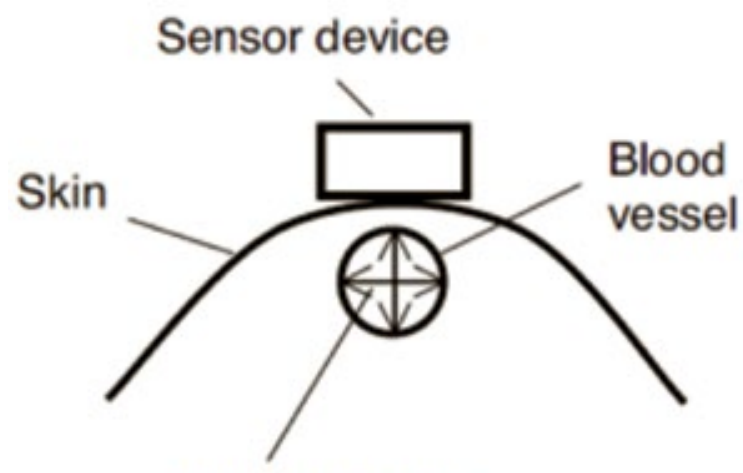

Overpressure
En este método de medición, la parte superior del vaso sanguíneo se hace plana, en la cual, la tensión del vaso sanguíneo funciona en ángulo recto a la dirección de detección de presión del sensor. La presión de la sangre, por tanto, se puede calcular directamente debido a que la fuerza medida por el sensor se hace igual a la presión de la sangre como se observa en la Fig. 12 (Dohi, 2014).

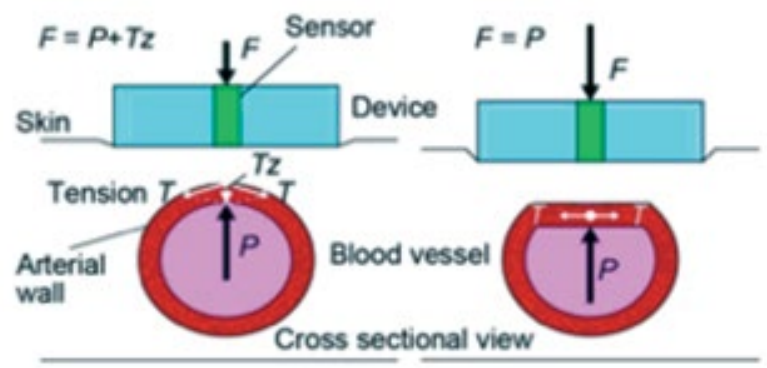

Fuente: (Dohi, 2014)

Fig. 12 (a) Arteria sin presión (b) Arteria con presión moderada Fuente: (Dohi, 2014)

El dispositivo de monitoreo se ubica en la muñeca y el sensor se debe pulsar sobre la arteria radial (Fig.13). Según la configuración del dispositivo, este emitirá una alerta sonora que indicará que se procederá a tomar una medición.

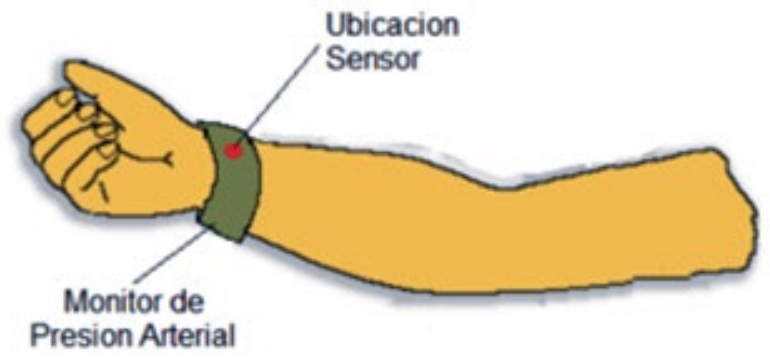

Fig. 13 Ubicación del dispositivo y el sensor. Fuente: autores

Fig. 11 Principio de monitoreo de presión arterial debido al desplazamiento de superficie

Fuente: (Kirstein et al., 2005) 


\subsubsection{Características técnicas}

del dispositivo final propuesto

En la Fig. 14 se presenta un diagrama de los bloques del dispositivo:

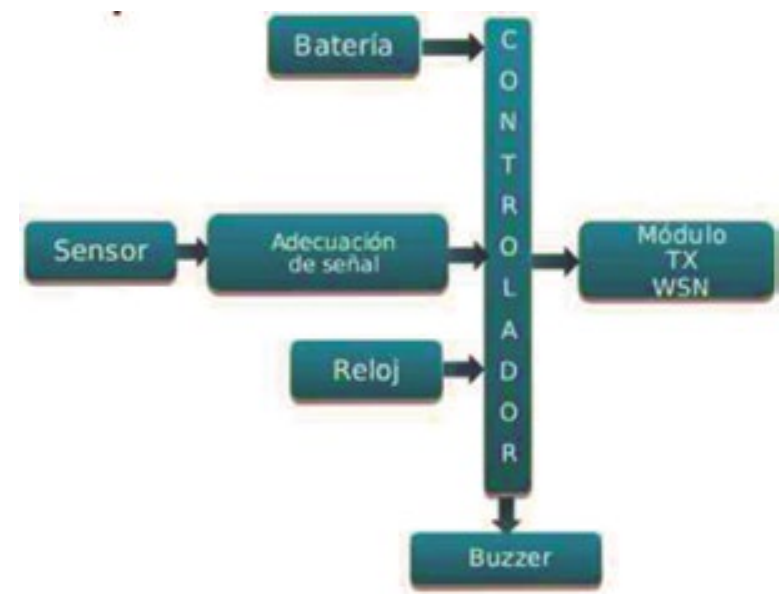

Fig. 14 Diagrama de Bloque Monitor Presión Arterial Fuente. autores

A continuación se hace una breve descripción de cada uno de los elementos que conforman este diagrama de bloque:

Batería: el dispositivo propuesto se alimenta por medio de una batería recargable de 3.6 voltios.

Sensor: el sensor por utilizar es un sensor de presión absoluta capacitivo, referencia Murata SCB10H.

Adecuación de señal: esta etapa se encarga de generar una seńal de voltaje proporcional a los cambios de capacitancia del sensor.

Reloj: se utiliza para realizar el control de tiempo al cual se deben tomar medidas de presión.

Controlador: este dispositivo es un microcontrolador PIC que realiza las siguientes funciones: emite una señal sonora que indica que se debe realizar una medida, procesa la información que recibe del sensor para obtener el valor de presión y envía estos valores al centro local de cómputo por medio del módulo de transmisión.

Buzzer: emite una señal sonora que indica que se debe realizar una medición de presión arterial.
Módulo TX WSN: este dispositivo es el encargado de enviar de forma inalámbrica los valores de presión arterial al centro local de cómputo. En este punto se pueden utilizar tres tipos diferentes de módulos, que se describen a continuación:

Módulo Bluetooth: permite realizar conexión con un teléfono inteligente que se encarga de enviar la información a la nube.

Módulo Wifi: permite la conexión del dispositivo con una red inalámbrica de área local.

Módulo Xbee: permite crear una red de dispositivos para tener acceso a su configuración.

\subsection{Desarrollo de la interfaz de software}

Como parte complementaria al dispositivo propuesto, el cual captura las señales biomédicas, se tiene la interfaz de software que en conjunto con el hardware se encarga de procesar la información de las pacientes relacionadas en el caso objeto de estudio. A continuación, se describen los elementos que componen esta parte del sistema.

\subsubsection{Administrador del sistema}

Para el monitoreo de las pacientes adscritas al caso de estudio propuesto, se desarrolló un sistema tipo Web, el cual, desde un servidor en la nube o de manera local, permite hacer seguimiento al proceso. Para efectos de esta investigación se utilizó un sitio gratuito cuya dirección es: http://preeclampsia.comxa.com/. En el sitio se puede monitorear en tiempo real a las pacientes con preeclampsia o en riesgo de esta enfermedad, que se han registrado previamente. El sistema permite el acceso a las pacientes y también el control por parte del personal médico que hace seguimiento a las mismas.

\subsubsection{Aspectos técnicos}

El software que compone el sistema de monitoreo, como se mencionó anteriormente, funciona bajo un servidor Web, lo que posibilita que se pueda manejar, monitorear y administrar desde plataformas que trabajan con internet o intranet cuando el servidor es de 
tipo local. Para el desarrollo de la investigación se utilizó un hosting gratuito, el cual permitió crear un piloto apropiado para recibir la información emanada por los sensores transmisores de las señales.

Como lenguaje de programación se utilizó PHP, que, de acuerdo con sus características y versatilidad, fue el más apropiado para el caso investigado. Es importante mencionar que junto al mismo se utilizaron herramientas de Java y Java-script. De la misma manera, se usó Mysql como motor de base de datos, para almacenar la información que envían los sensores, el cual trabajó de manera adecuada con el lenguaje PHP; además, su portabilidad hace que se pueda utilizar en diversos tipos de navegadores tanto para PC, tablets y móviles. La base de datos contiene las tablas paciente, personal y sesión que cumplen las siguientes funciones:

Tabla paciente: es la tabla maestra del sistema. En la misma se almacena la información de las pacientes, emitida por los sensores que poseen las usuarias del sistema.

Tabla personal: es la tabla de registro de información básica del personal médico, que, para efectos del caso de estudio, es el que está al pendiente del monitoreo de las pacientes que hacen uso de los sensores.

Tabla sesión: es una tabla transitoria que controla el registro de pacientes y personal médico en el sistema; actúa como un control de acceso al sistema, para mantener la seguridad del mismo.

Es importante mencionar en estos aspectos técnicos que al utilizar el servicio en la nube de hosting, la aplicación para el seguimiento de pacientes con preeclampsia se hace en tiempo real, debido a que la actualización de la base de datos de la información que capturan los sensores de las usuarias se realiza directamente en la nube o centro de cómputo, permitiendo que los datos estén disponibles en el momento que sean requeridos.

\subsubsection{Descripción del software piloto:}

el software piloto para el monitoreo de pacientes diagnosticadas con preeclampsia presenta los siguientes módulos:
Página principal: incluye el módulo de acceso para pacientes, personal médico y asistencia.

El módulo pacientes permite que se registre a las usuarias del sistema con el número de identificación de las mismas y que este sea comparado con los registros que se envían periódicamente por los sensores que tenga asignados la paciente. El registro e ingreso de la paciente a este módulo, le permite observar la medición de su presión arterial, permitiendo identificar los estados de alerta con respecto a la evolución de su patología, así como al personal médico que está realizando el seguimiento Fig.15.

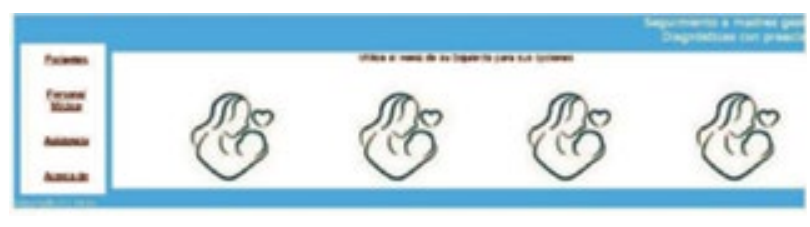

Fig. 15 Página principal

Fuente: http://preeclampsia.comxa.com/

Página pacientes: esta página permite a las pacientes monitorear la lectura que hacen los sensores de su presión arterial, la cual puede presentar tres estados: alerta baja o normal, media o leve y alta o grave.

- Alerta baja o normal: la prueba inicial muestra que la paciente tiene una presión arterial menor o igual a $120 \mathrm{mmHg}$ que, de acuerdo con los estudios previos del proyecto, muestran que la presión arterial sistólica es la que indica el factor de riesgo bajo para la paciente gestante.

- Alerta media o leve: el estado medio se da cuando los rangos de presión oscilan entre los 121 $\mathrm{mmHg}$ hasta los $135 \mathrm{mmHg}$. En este estado la presión indica que la paciente pudo tener algún evento físico transitorio en el cual su presión arterial alta se vio afectada. Este estado dependerá de las lecturas siguientes ya que la presión se irá registrando en la base de datos y la usuaria como el personal médico deben estar pendientes de la evolución, en caso de pasar al siguiente nivel de alerta. 
- Alerta alta o grave: a esta condición que puede presentar una paciente se denomina de atención inmediata - alta - grave, dado que la presión arterial supera los $150 \mathrm{mmHg}$, que de acuerdo con la descripción de los valores de cuidado antes vistos, es una condición de urgencia, en la cual puede haber complicaciones, que afecten tanto a la paciente como al feto Fig.16.

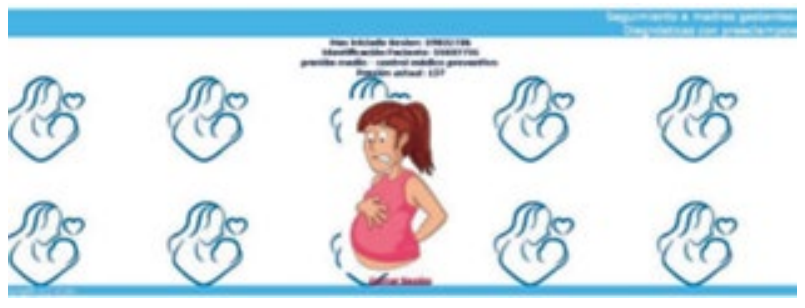

Fig. 16 Alerta alta.

Fuente: http://preeclampsia.comxa.com/

Página de personal médico: la página de personal médico permite el ingreso a los usuarios que realizan el seguimiento a las pacientes, quienes son los encargados de observar la condición de las mismas y serán quienes determinen las acciones por realizar de acuerdo con las alertas que se observen y el protocolo médico que se tiene para cada caso Fig.17.

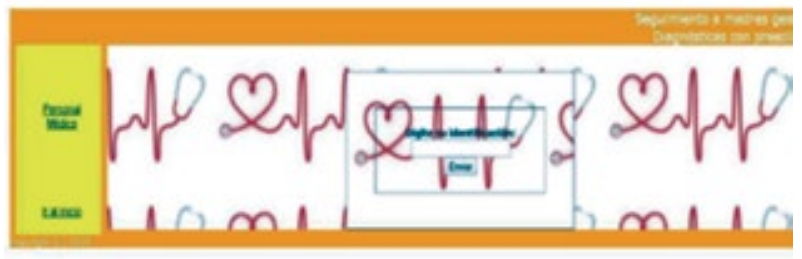

Fig. 17 Ingreso personal médico

Fuente: http://preeclampsia.comxa.com/

Las opciones que están disponibles en esta versión piloto del software son: madres online, en la que se puede identificar las pacientes que están registradas y con los sensores activos Fig.18, y el informe y resumen de seguimiento, en el que el personal médico puede descargar un documento en formato PDF con el resumen total de mediciones de la presión arterial de las pacientes diagnosticadas con preeclampsia, el cual sirve como registro histórico para el personal médico Figuras 19 y 20.

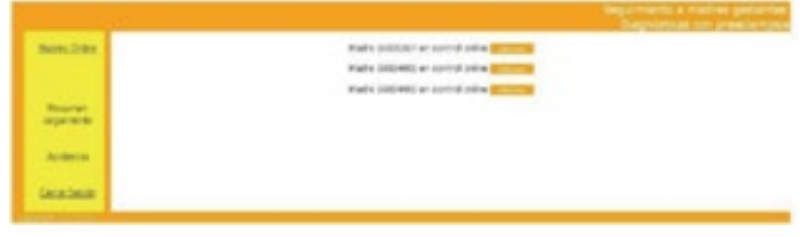

Fig. 18 Madres online

Fuente: http://preeclampsia.comxa.com/

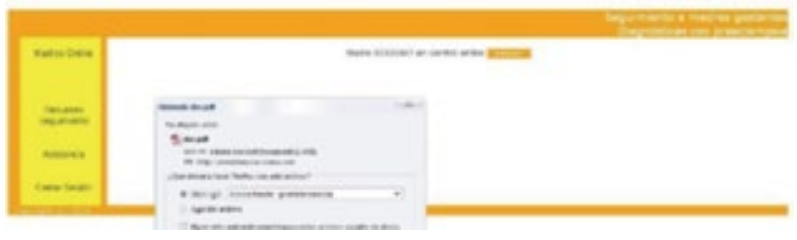

Fig. 19 Página informes

Fuente: http://preeclampsia.comxa.com/

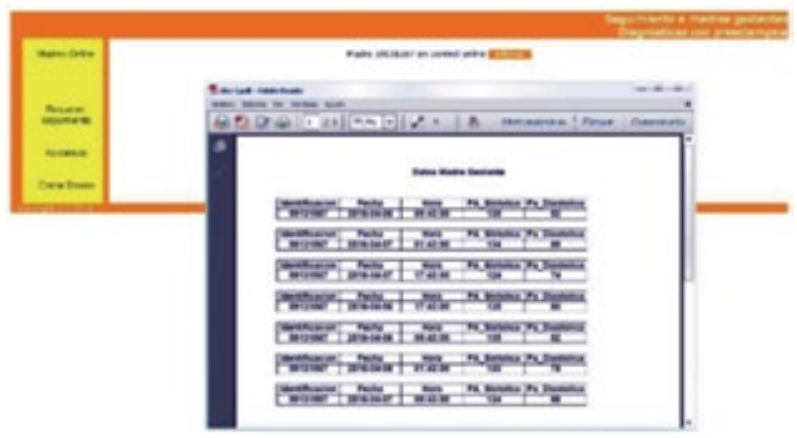

Fig. 20 PDF informes seguimiento

Fuente: http://preeclampsia.comxa.com/

4.4.4. Reportes y lógica difusa en el sistema propuesto Otro elemento importante que se abordó en la investigación realizada, fue la generación de reportes, los cuales se buscó que fueran de fácil lectura para las usuarias y el personal médico a cargo. En este aspecto, se incursionó en el desarrollo de un sistema de reportes difuso, el cual tuvo en cuenta los datos que capturaron los sensores y la forma en que se interpretaron.

Enfoque difuso: de acuerdo con Castillo \& Trejos (2009), el modelado lingüístico difuso es una herramienta que hace posible la representación de aspectos cualitativos, basada en el concepto de variables lingüísticas, que son palabras o sentencias expresadas en lenguaje natural o artificial. Es decir, se interpreta el fenómeno o actividad, 
el cual puede darse en valores numéricos, pero expresados mediante sentencias de texto más comprensibles para el usuario final. En otras palabras, gracias al enfoque difuso es posible interpretar un contexto de una forma más flexible que la lógica tradicional, en la que los valores son absolutos y rígidos, pasando a un esquema en el que la variabilidad y las diferencias mínimas o máximas pueden ser tenidas en cuenta. Con respecto al sistema desarrollado, este enfoque permite determinar los estados que se mencionaron anteriormente en la descripción de la interfaz de software (alerta baja o normal, media o leve y alta o grave) y hacen que el monitoreo sea más ajustado a la realidad sin generar alertas que en un determinado momento indican acciones que no se deben desarrollar.

Las variables médicas, los sensores y el reporte de software en lógica difusa: en la investigación desarrollada y acorde con lo explicado anteriormente se pudo identificar que la variable principal por monitorear es la presión arterial. Con base en este aspecto, se identificaron los siguientes elementos con respecto a los reportes diseñados con lógica difusa:

- Variables lingüísticas de entrada: presión arterial PAS, que corresponde a la presión arterial sistólica y presión arterial PAD, equivalente a presión arterial diastólica. En estas dos variables se tuvo en cuenta los valores desde el nivel normal hasta los que se consideran altos. Para este caso de estudio y patología seleccionada, no se tuvieron en cuenta los valores bajos, por cuanto corresponden a otro tipo de enfermedades. En las figuras 21 y 22 se pueden observar los rangos de las variables lingüísticas definidas.

Para la presión arterial - sistólica (PAS): se tuvo como rango los valores entre 120 y $220 \mathrm{mmHg}$, los cuales generaron los siguientes valores difusos: normal, anormal leve y anormal grave.

Para la presión arterial - diastólica (PAD): el rango estuvo entre 80 y $120 \mathrm{mmHg}$, los cuales generaron los siguientes valores difusos: normal, anormal leve y anormal grave.

\section{PAS Normal, Leve y Grave}

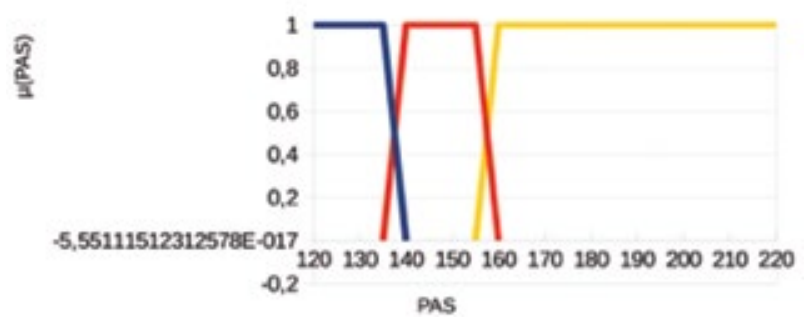

Fig. 21 Presión arterial sistólica valores difusos

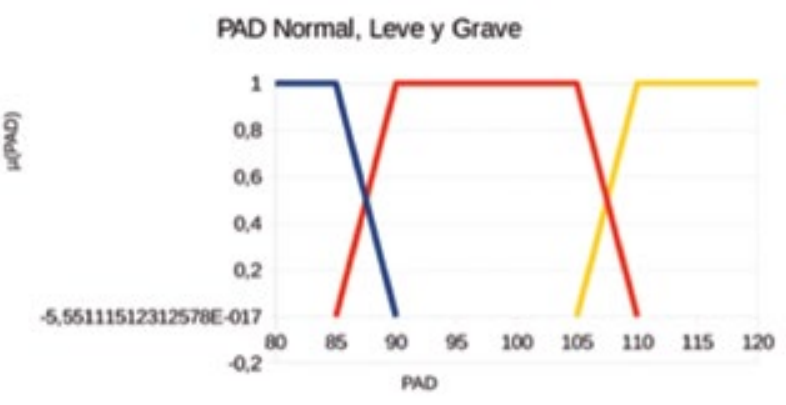

Fig. 22 Presión arterial diastólica valores difusos Fuente: E. Nummelin, 2015.

- Variable de salida: acorde con las variables de entrada que corresponden a los valores de la presión arterial PAS y PAD, se tiene como variable de salida el posible diagnóstico de la paciente (DP), el cual podrá tener los siguientes valores lingüísticos: ausencia de preeclampsia, posible preeclampsia leve, posible preeclampsia grave. Estas variables se conjugan con las de entrada mediante la herramienta que brinda la lógica difusa y se denominan "Reglas difusas", las cuales se mencionan a continuación.

- Reglas difusas: a partir de algunos protocolos médicos (Gutiérrez et al., 2002), que definen la forma de identificar y diagnosticar la preeclampsia, y también de otros estudios relacionados con el manejo de la misma y las variables asociadas (Artalejo \& Ortún, 1990; Wilbik, Keller y Alexander, 2011; Zadeh, 1975), se definieron las reglas difusas que permiten generar los reportes en el sistema tabla 6 . 


\section{TABLA 6}

Reglas difusas para el sistema de monitoreo

\begin{tabular}{|c|c|c|}
\hline Regla & Antecedente & Consecuente \\
\hline 1 & $\begin{array}{l}\text { SI (PAS es normal) } \\
\text { OR (PAD } \\
\text { es normal) }\end{array}$ & $\begin{array}{l}\text { Entonces (DP es ausencia } \\
\text { de preeclampsia) }\end{array}$ \\
\hline 2 & $\begin{array}{l}\text { SI (PAS es anormal } \\
\text { leve) OR (PAD } \\
\text { es normal) }\end{array}$ & $\begin{array}{l}\text { Entonces (DP es posible } \\
\text { preeclampsia leve) }\end{array}$ \\
\hline 3 & $\begin{array}{l}\text { SI (PAS es anormal } \\
\text { leve) OR (PAD } \\
\text { es anormal leve) }\end{array}$ & $\begin{array}{l}\text { Entonces (DP es posible } \\
\text { preeclampsia leve) }\end{array}$ \\
\hline 4 & $\begin{array}{l}\text { SI (PAS es normal) } \\
\text { OR (PAD es } \\
\text { anormal leve) }\end{array}$ & $\begin{array}{l}\text { Entonces (DP es posible } \\
\text { preeclampsia leve) }\end{array}$ \\
\hline 5 & $\begin{array}{l}\text { SI (PAS es anormal } \\
\text { grave) OR (PAD } \\
\text { es normal) }\end{array}$ & $\begin{array}{l}\text { ENTONCES (DP es po- } \\
\text { sible preeclampsia grave) }\end{array}$ \\
\hline 6 & $\begin{array}{l}\text { SI (PAS es anormal } \\
\text { grave) OR (PAD } \\
\text { es anormal grave) }\end{array}$ & $\begin{array}{l}\text { (Entonces DP es posible } \\
\text { preeclampsia grave) }\end{array}$ \\
\hline 7 & $\begin{array}{l}\text { SI (PAS es normal) } \\
\text { OR (PAD } \\
\text { es anormal grave) }\end{array}$ & $\begin{array}{l}\text { Entonces (DP es posible } \\
\text { preeclampsia grave) }\end{array}$ \\
\hline
\end{tabular}

La explicación del modelo difuso y los reportes que se generaron son tema de otro artículo, debido a la cantidad de elementos que se deben describir y detallar para que el lector pueda comprenderlo. No obstante, a nivel general, en este apartado se ha querido mencionar la forma en que se generaron las alertas y los controles que se tuvieron en cuenta para los reportes que se deben entregar a las usuarias del sistema y el personal médico a cargo.

\section{Conclusiones y trabajo Futuro}

La preeclampsia se constituye como una patología de alto riesgo en mujeres gestantes, sobre todo a partir del tercer trimestre de su embarazo; por ello, hacer todos los esfuerzos en pro de su cuidado es vital para evitar desenlaces fatales.

El cuidado de la salud es un campo con mayor aplicación tecnológica y todo avance y propuesta que coadyuve en su desarrollo no está de más; por el contrario, se deben potenciar las posibilidades de nuevas y mejores formas de ayuda y control de enfermedades asociadas con el riesgo de muerte en el hombre.

Las redes WSN asociadas al cuidado de la salud son una tecnología emergente que aún tiene mucho por investigar y desarrollar en pro de nuevas posibilidades que permitan un mayor control y seguimiento a enfermedades, como el caso de la preeclampsia.

En el desarrollo del sistema de monitoreo propuesto, el uso de un servidor Web en la nube permite lecturas y almacenamiento en línea de datos, lo cual posibilita que se pueda obtener información oportuna para las usuarias y el personal médico a cargo, así como también, facilitar la aplicación de procesos de minería de datos que complementen el estudio propuesto.

La creación de alertas para pacientes y personal médico desde la información residente en el servidor, se flexibiliza porque el medio por donde se puede entregar se amplía a dispositivos de comunicaciones diversos.

La interpretación correcta de los resultados médicos es tan importante como la consecución de los mismos, puesto que el tratamiento del paciente depende de los resultados de este análisis. En estos casos, los modelos difusos proporcionan una ayuda fundamental en el logro de este proceso.

La lógica difusa es una técnica ampliamente investigada que se puede adaptar a muchas áreas de aplicación. En el caso de seguimiento y análisis de señales biomédicas, sigue siendo un reto para establecer un modelo general que traduzca los resultados obtenidos por los sensores en resúmenes lingüísticos.

Como trabajos futuros se proyecta el desarrollo de un sistema inteligente para generar resúmenes lingüísticos en el monitoreo de señales biomédicas, principalmente para el manejo de las variables de entrada en función del tiempo y la identificación de patrones en los pacientes que padecen la misma patología, con el apoyo adicional de un experto en el campo para la definición de los conjuntos difusos de salida. 
También se proyecta la implementación del prototipo del sistema desarrollado en esta investigación y el mejoramiento del mismo para que pueda convertirse en un aliado en el control de una enfermedad tan temida como la preeclampsia.

\section{REFERENCIAS}

Abbate, S, Avvenuti, M \& Light, J 2012, 'Mims: a minimally invasive monitoring sensor platform', Sensors Journal Ieee, 12(3), 677-684.

Akyildiz, I, Su, W, Sankarasubramaniam, Y \& Cayirci, E 2002, 'Asurvey on sensor networks'. Communications magazine Ieee, 40(8), 102-114.

Bravo, O, Valdez, E \& Medina, A 2015, 'Monitoreo inalámbrico de un electrocardiógrafo', 21 Congreso Internacional Mexicano de Acústica, Universidad de Guadalajara, Centro Universitario de Ciencias Biológicas y Agropecuarias, León, México.

Castillo, W \& Trejos, J 2009, 'Propiedades de un enfoque difusopara particionamiento con datos bimodales', Revista de matemática: Teoría y aplicaciones, 16 (2), 241-254.

Chaithanya, M, Kishore, K \& Srinivasulu, A 2014, 'ContinuesBlood Pressure Measurement and Data Logging Device with SMS Alert', International Journal of Multimedia and Ubiquitous Engineering, 9 (8), 25-40.

Chen, X, Lu, X, Liu, Z, Fang, S, Jin D \& Zeng, L 2011, 'A heterogeneous high speed wireless body sensor network based on sc-uwb and zigbee'. 2011 Global Telecommunications Conference (GLOBECOM 2011), IEEE, Houston, pp. 1-5.

Dohi, T 2014, 'Measuring blood pressure with mems' [en línea], The Japan News, s.f, consultado: Marzo 2016 http://www.yomiuri.co.jp/adv/chuo/dy/opinion/20150511.html

Godoy, D 2010, 'Proyecto Diseño y construcción de una plataforma de telemedicina para el monitoreo de bioseńales', SenaColciencias.

González, A., Núñez, M \& García, P 2009, 'Diseño de un simulador para redes de sensores, trabajo diseñado para el curso sistemas informáticos', Universidad Complutense de Madrid, España.

Gutiérrez, A, Herraiz, M, Bellon, M, Martell, N \& Escudero, M2002, 'Protocolo de actuación ante la preeclampsia. Nuestra experiencia', Revista actualidad Obstétrico Ginecológica. Departamento de Obstetricia y Ginecologia,XIV (1), 7-14.

Hipertensión y embarazo: preeclampsia - eclampsia, (s.f.) [Páginaweb], consultado: abril 2016, http://www.intermedicina. com/Estudiantil/Novedades/Nov11.htm

Kaschel, H \& Pérez, J 2014, 'Monitoreo ubicuo de salud en tiemporeal con Wbsn', Revista Chilena de Ingeniería, 22(2), 169-176.
Kappiarukudil, K \& Ramesh, M 2010, 'Real-time monitoring and detection of" heart attack" using wireless sensor networks', 2010 Sensor Technologies and Applications (Sensorcomm), Fourth International Conference On, Ieee, Venecia, pp. 632-636.

Kirstein, K, Sedivy, J, Salo, T, Hagleitner, C, Vancura, T \& Hierlemann, A 2005, 'A CMOS-based Tactile Sensor for Continuous Blood Pressure Monitoring', 2005 IEEE Proceedings of the Design, Automation and Test in Europe Conference and Exhibition, IEEE, Munich.

López, S 2012, Blood Pressure Monitor Fundamentals and Design[en línea], consultado Abril 2016: https://www.nxp. com/docs/en/application-note/AN4328.pdf

Luna, J 2015, Preeclamsia [Página web], consultado marzo 2016: Available: http://www.infosalus.com/patologia-preeclampsia-102.html

Martínez, D, Blanes, F, Simo, J \& Crespo, A 2008, 'Redes de sensores y actuadores inalámbricas: una caracterización y caso de estudio para aplicaciones médicas en espacios cerrados', XXIX Jornadas de Automática, Universidad Rovira i Virgili de Tarragona, España.

Mohammadi, V, Torkian, S, Masumi, E, Sheikhi, M, Barzegar A \& Mohammadi, S 2009, 'Design, Modeling and Optimization of a Piezoelectric Pressure Sensor Based On Thin-Film PZT Diaphragm Contain of Nanocrystalline Powders', 2009 IEEE International Symposium on Mechatronics and its Applications, IEEE, Malaga.

Moine M 2011, 'Estudio comparativo de metodologías para minería de datos'. Trabajo presentado en XIII Workshop de Investigadores en Ciencias de la Computación, consultado abril 2016 :http://sedici.unlp.edu.ar/bitstream/handle/10915/20034/Documento_completo.pdf\%3Fsequence $\% 3 \mathrm{D} 1$

Morparia, K, Shah, P, Shah B \& Krishnamachari, B 2007, 'Experimental study of concurrent packet transmission in wireless sensor networks using tmote sky devices', SenSys'06: Proceedings of the Fourth International Conference on Embedded Networked Sensor Systems, ACM, Boulder, Colorado, pp. 237-250

Nummelin, E 2015, 'Murata - a pressure sensor based bloodpressure monitor, tesis de pregrado', Helsinki Metropolia University of Applied Science, Helsinki, Finlandia.

Park, J \& Zheng, G 2011, 'An emergency-adaptive routing scheme for wireless sensor networks for building fire hazard monitoring', Sensors, 11(3), 2899-2919.

Rathore, P \& Panwar, B 2013, 'Design and Optimization of a CMOS_MEMS Integrated Current Mirror Sensing Based MOSFET Embedded Pressure Sensor', 2013 Ieee International Conference on Control Applications, Ieee, Hyderabad, pp. 442-448.

Rodríguez Artalejo F \& Ortún Rubio V 1990, Los protocolos clínicos. Med Clin (Barc), 95:309-316.

Rojas, J 2009, Protocolo de manejo de Preeclampsia - Eclampsia, Clínica de Maternidad Rafael Calvo, Cartagena. 
Saavedra, H, Aguirre, J, Mejía, A, Riaño, K, Romero, C, Sierra, C, Angulo F \& Márquez, L 2014, 'Diseño e implementación de un prototipo para automatización de vivienda', TC. Ingeniería Electrónica.

Shahiri, M, Ganji, B \& Sabbaghi, R 2012, 'Design and Simulation of High Sensitive Capacitive Pressure Sensor with Slotted Diaphragm', 2012 IEEE International Conference on Biomedical Engineering, IEEE, Langkawi, Malasia, pp. 484-489.

Shahriyar, R, Bari, M, Kundu, G, Ahamed, S \& Akbar, M 2009, 'Intelligent mobile health monitoring system (imhms)', Electronic Healthcare, 27, 5-12.

Wilbik, A, Keller, J \& Alexander, G 2011, 'Linguistic summarization of sensor data for eldercare', 2011 IEEE International Conference Systems, Man, and Cybernetics (SMC), IEEE, Anchorage, pp. 2595-2599.

Witten, I \& Eibe, F 2005, Data Mining: Practical machine learning tools and techniques, segunda edición, Morgan Kaufmann, San Francisco.
Yang, C \& Huang, T 2012, 'The era of cloud computer: Thru biodetecting and open-resources to achieve ubiquitous devices', 2012 International Symposium Computer, Consumer and Control (IS3C), , IEEE, Taichung, Taiwan, pp. 8-11.

Yunus, N, Halin, I, Sulaiman, N, Ismail, N \& Sheng, O 2015, 'Valuation on MEMS Pressure Sensors and Device Applications', Journal of Electrical, Computer, Energetic, Electronic and Communication Engineering, 9 (8), 834842.

Zadeh, L 1975, 'The concept of a linguistic variable and its applications to approximate reasoning', Informations sciences, 8(3), 199-249.

Zeng, Y, Sreenan, C, Sitanayah, L, Xiong, N 2011, 'An emergencyadaptive routing scheme for wireless sensor networks for building fire hazard monitoring' Sensors, 11(3), 2899-2919. 
Revista Especializada en Ciencia, Tecnología e Ingeniería 
